Laurence Vignaux

Frédéric Vargas

Jean Roeseler

Didier Tassaux

Arnaud W. Thille

Michel P. Kossowsky

Laurent Brochard

Philippe Jolliet

\section{Patient-ventilator asynchrony during non-invasive ventilation for acute respiratory failure: a multicenter study}

Received: 13 May 2008

Accepted: 12 December 2008

Published online: 29 January 2009

(C) Springer-Verlag 2009

This study was presented in abstract form at the 2008 Annual Meeting of the American Thoracic Society.

Electronic supplementary material The online version of this article (doi:10.1007/s00134-009-1416-5) contains supplementary material, which is available to authorized users.

L. Vignaux · D. Tassaux · P. Jolliet Service des Soins Intensifs, Hôpitaux universitaires de Genève, Geneva, Switzerland

F. Vargas · A. W. Thille · L. Brochard Réanimation Médicale, AP-HP, Centre Hospitalier Albert ChenevierHenri-Mondor, Créteil, France

F. Vargas - A. W. Thille - L. Brochard INSERM U 841, Créteil, France

F. Vargas - A. W. Thille - L. Brochard Université Paris 12, Créteil, France

J. Roeseler

Service des Soins Intensifs, Cliniques

Universitaires St.-Luc, Brussels, Belgium
M. P. Kossowsky

Service de Médecine de Premier Recours, Hôpitaux universitaires de Genève, Geneva, Switzerland

\section{P. Jolliet ( $)$}

Service des Soins Intensifs, Hôpital

Cantonal Universitaire, 1211 Geneva 14,

Switzerland

e-mail: jolliet@medecine.unige.ch

Tel.: +41-22-3729093

Fax: +41-22-3729105

Abstract Objective: To determine the prevalence of patient-ventilator asynchrony in patients receiving noninvasive ventilation (NIV) for acute respiratory failure. Design: Prospective multicenter observation study. Setting: Intensive care units in three university hospitals. Methods: Patients consecutively admitted to ICU were included. NIV, performed with an ICU ventilator, was set by the clinician. Airway pressure, flow, and surface diaphragmatic electromyography were recorded continuously for $30 \mathrm{~min}$. Asynchrony events and the asynchrony index (AI) were determined from visual inspection of the recordings and clinical observation. Results: A total of 60 patients were included, $55 \%$ of whom were hypercapnic. Auto-triggering was present in
$8(13 \%)$ patients, double triggering in 9 (15\%), ineffective breaths in $8(13 \%)$, premature cycling $7(12 \%)$ and late cycling in $14(23 \%)$. An $\mathrm{AI}>10 \%$, indicating severe asynchrony, was present in 26 patients (43\%), whose median (25-75 IQR) AI was 26 (15-54\%). A significant correlation was found between the magnitude of leaks and the number of ineffective breaths and severity of delayed cycling. Multivariate analysis indicated that the level of pressure support and the magnitude of leaks were weakly, albeit significantly, associated with an AI > 10\%. Patient comfort scale was higher in pts with an $\mathrm{AI}<10 \%$. Conclusion: Patientventilator asynchrony is common in patients receiving NIV for acute respiratory failure. Our results suggest that leaks play a major role in generating patient-ventilator asynchrony and discomfort, and point the way to further research to determine if ventilator functions designed to cope with leaks can reduce asynchrony in the clinical setting.

Keywords Noninvasive ventilation . Mechanical ventilation .

Patient-ventilator interaction . Asynchrony 


\section{Introduction}

Non-invasive ventilation (NIV) has become a standard of care in both hypercapnic and non-hypercapnic acute respiratory failure [1-3]. However, patient tolerance to the technique is a critical factor determining its success in avoiding endotracheal intubation [4]. One of the key factors determining tolerance to NIV is optimal synchrony between the patient's spontaneous breathing activity and the ventilator's set parameters, known as "patient-ventilator interaction" [5,6]. Optimal patientventilator synchrony can prove very difficult to achieve, especially during NIV, due to the presence of leaks at the patient-mask interface which can interfere with various aspects of ventilator function [7-9]. This interference can increase the risk of asynchrony, which in turn leads to an increase in the work of breathing (WOB) and patient discomfort $[5,6]$.

A recent study in intubated patients undergoing pressure support ventilation (PSV) has shown that $25 \%$ of these patients exhibited a high degree of asynchrony, the latter being associated with a prolonged duration of mechanical ventilation [10]. To date, however, no such data are available in patients during NIV. Therefore, the goal of the present multicenter study was to document the nature and severity of patient-ventilator asynchrony during NIV in the setting of acute respiratory failure.

\section{Materials and methods}

This prospective, observational study was conducted in three university hospital ICUs, one medical (Créteil) and two medical-surgical (Brussels, Geneva). The sample size was arbitrarily set at 60 patients, included as soon as possible after ICU admission. The protocol was approved by the Ethics Committee of each participating center. All patients with acute hypercapnic or non-hypercapnic respiratory failure, admitted to the ICU for NIV, were eligible. Informed consent was obtained from the patients.

All patients were ventilated with an ICU ventilator, the type depending on availability and center. Criteria for initiating NIV followed the usual practice guidelines of the participating centers, which are based on published studies on hypercapnic [11] and non-hypercapnic [3] respiratory failure (see ESM). Patients were excluded from receiving NIV if any of classical contraindications to its application were present [12].

The NIV settings were made by the clinician in charge of the patient. NIV was applied with an oro-nasal mask, in PSV mode, as per the standard procedures in the centers. No intervention whatsoever was made by the investigators.
Measured parameters

The respiratory parameters and asynchrony events were assessed by analysis of the flow, pressure, and surface diaphragmatic electromyographic activity (EMGd) signals, based on the methodology by Thille et al. [10], to which EMGd recording was added (see ESM).

Asynchrony events (Ineffective triggering, doubletriggering, auto-triggering, premature cycling, and delayed cycling) were detected by visual inspection of the recordings (Fig. 1), as detailed in the ESM.

A global asynchrony index (AI) was computed as previously published $[10,13]$. An AI $>10 \%$ was considered as severe $[10,13]$. Comfort was assessed using a visual analog scale ( 0 worst possible to 10 best possible sensation of overall comfort during NIV).

\section{Statistics}

Statistics were computed with the STATA 10.0 Statistics/ Data analysis package (Stata Corp., College Station, TX, USA). Tests are detailed in the ESM.

\section{Results}

Sixty-eight patients were screened, three of whom refused to consent. Sixty-five patients were therefore included, five of whom were excluded due to technical issues precluding an adequate analysis of the tracing. A total of 60 patients were therefore finally included in the study, whose main characteristics and outcome parameters are outlined in Tables 1 and 2. Only 23\% of patients presented with no chronic cardio-respiratory problem. The most frequently documented chronic conditions were COPD $(40 \%)$, heart failure (28\%), obesity-hypoventilation $(15 \%)$, restrictive disease $(7 \%)$, neuromuscular disease $(5 \%)$, and mixed obstructive/restrictive problems (3\%). Upon study inclusion, the patients had received $4 \pm 2$ prior sessions of NIV. Inclusion took place 1.3 $( \pm 0.2)$ days after the initial NIV session. Thirty-three patients $(55 \%)$ were hypercapnic $\left(\mathrm{PaCO}_{2}>42 \mathrm{mmHg}\right.$ or $5.6 \mathrm{kPa}$ ). The ventilator settings and respiratory parameters are summarized in Table 3 . Of note, expiratory trigger setting was the default for all patients, i.e., 25 or $30 \%$ of peak inspiratory flow depending on the ventilator used. Characteristic recordings of the various asynchrony events are shown in Fig. 1. Auto-triggering was present in $8(13 \%)$ patients, double triggering in $9(15 \%)$, ineffective breaths in $8(13 \%)$, premature cycling $7(12 \%)$ and late cycling in 14 (23\%). Eighteen patients presented with only one type of asynchrony, ten patients had 2 types and four had 3 types. Ineffective breaths and delayed cycling 
Fig. 1 Representative tracings of the five types of asynchrony. $E M G_{d i}$ diaphragmatic

electromyography tracing; Paw airway pressure; $V$ '

instantaneous flow. Downward pointing arrows indicate relevant event

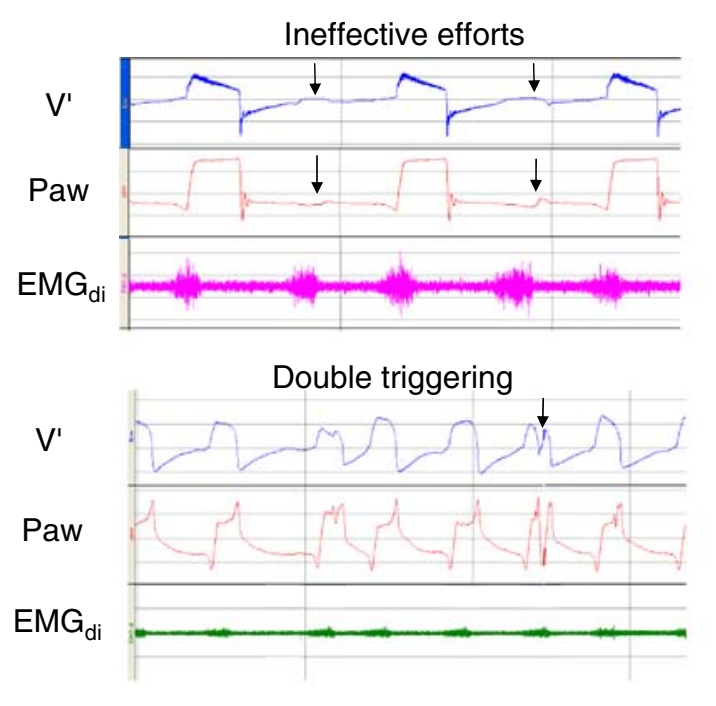

Late cycling and in effective triggering

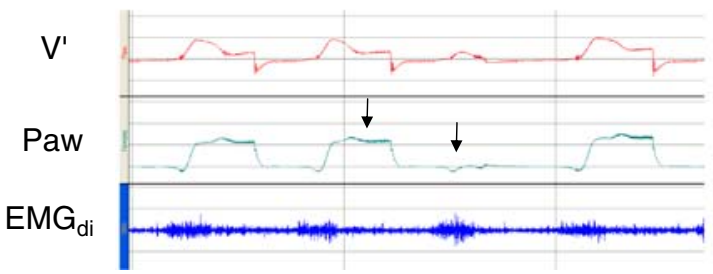

Auto triggering

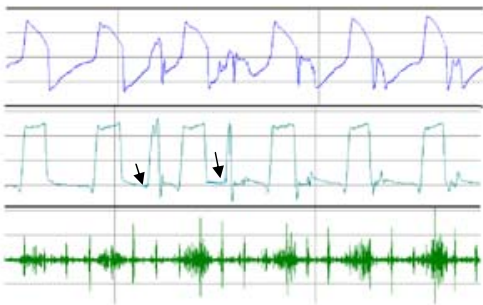

Premature cycling

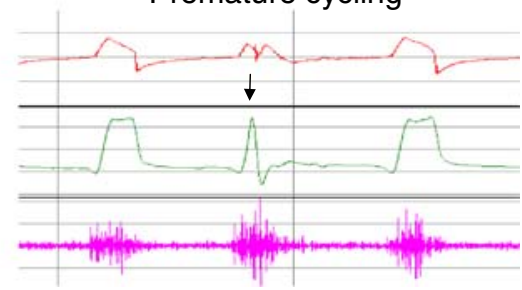

were the only two asynchronies associated likely to be grouped $(P=0.003)$. An $\mathrm{AI}>10 \%$, indicating severe asynchrony, was present in 26 patients $(43 \%)$, in whom the median (IQR) AI was $26(15-54) \%$. In the random sample of 21 patients, the number of ineffective breaths and auto-triggering events detected clinically and from tracings were compared and found to be statistically not different $(P=0.81)$. Table 4 lists the respiratory parameters recorded in patients with and without the

Table 1 Main clinical characteristics of the patients $(n=60)$

\begin{tabular}{lc}
\hline Demographics and initial respiratory parameters mean (SD) \\
Age (year) & $70(12)$ \\
$\mathrm{M}: \mathrm{F}$ & $41: 19$ \\
$\mathrm{BMI}\left(\mathrm{kg} / \mathrm{m}^{2}\right)$ & $26(7)$ \\
$\mathrm{SAPS} \mathrm{II}$ & $43(15)$ \\
$\mathrm{RR}(\mathrm{n} / \mathrm{min})$ & $29(7)$ \\
$\mathrm{PaO}_{2} / \mathrm{FIO}$ & $186(83)$ \\
$\mathrm{PaCO}_{2}(\mathrm{mmHg})$ & $48(16)$ \\
$\mathrm{Cause}_{2}$ of acute respiratory failure $n(\%)$ & \\
Acute on chronic respiratory failure & $25(38)$ \\
Community-acquired pneumonia & $19(20)$ \\
Post-extubation & $15(25)$ \\
Cardiogenic pulmonary edema & $9(25)$ \\
Post-operative & $4(7)$ \\
Thoracic trauma & $3(5)$
\end{tabular}

$B M I$ body mass index; $M: F$ male:female ratio; SAPS $I I$ admission Simplified Acute Physiologic Score; $R R$ respiratory rate various asynchrony events, indicating the significant differences, if any, between these two conditions. Parameters not shown in Table 4 exhibited no significant difference between presence and absence of any of the asynchrony events. No difference was noted between the groups in terms of cause of acute respiratory failure, except for pneumonia which was more prevalent in patients with premature cycling (14 vs. 5 patients, $P<0.028)$. Linear regression analysis showed that the magnitude of leak was significantly associated with the number of ineffective breaths (increase of mean leak flow of $0.67 \mathrm{l} / \mathrm{min}$ per one additional ineffective breath, $P=0.006$ ) and the presence of delayed cycling (increase of mean leak flow of $3.3 \mathrm{l} / \mathrm{min}$ if delayed cycling was present vs. no delayed cycling, $P=0.005)$. No

Table 2 Main outcome parameters $(n=60$ pts)

\begin{tabular}{lc}
\hline Number of days during which NIV was applied & $4.8(3)$ \\
Number of NIV sessions/day during ICU stay & $5(1.6)$ \\
Duration of NIV sessions (min) & $59(48)$ \\
Endotracheal intubation $(n$ pts $(\%))$ & $12(20)$ \\
ICU mortality ( $n$ pts $(\%))$ & $6(10)$ \\
LOS in ICU (days) & $10(5.8-17)$ \\
LOS in hospital (days) & $21(16-45)$
\end{tabular}

All values mean (SD) or median (25-75 interquartile range) $N I V$ noninvasive ventilation; $L O S$ length of stay in ICU or hospital 
Table 3 Ventilator settings and respiratory parameters during NIV $(n=60 \mathrm{pts})$

\begin{tabular}{|c|c|}
\hline \multicolumn{2}{|l|}{ Ventilator settings } \\
\hline PSL $\left(\mathrm{cmH}_{2} \mathrm{O}\right)$ & $11(3-20)$ \\
\hline PEEP $\left(\mathrm{cmH}_{2} \mathrm{O}\right)$ & $5.5(2-8)$ \\
\hline Pressurization slope (ms) & $190(50-400)$ \\
\hline \multicolumn{2}{|l|}{ Inspiratory trigger } \\
\hline Flow $(n=57)(1 / \mathrm{min})$ & $1.1(0.3-3)$ \\
\hline Pressure $(n=3)\left(\mathrm{cmH}_{2} \mathrm{O}\right)$ & $2-3$ \\
\hline Expiratory trigger ${ }^{\mathrm{a}}(\%)$ & $25-30$ \\
\hline $\mathrm{FIO}_{2}$ & $0.45(0.1)$ \\
\hline \multicolumn{2}{|c|}{ Respiratory parameters while on NIV } \\
\hline $\operatorname{RR}(n / \min )$ & $25(6)$ \\
\hline $\mathrm{ti}_{\mathrm{p}}(\mathrm{ms})$ & $793(310-2,770)$ \\
\hline $\mathrm{ti}_{\text {excess }}\left(\%\right.$ of $\left.\mathrm{ti}_{\mathrm{p}}\right)$ & $37(-36$ to +140$)$ \\
\hline \multicolumn{2}{|l|}{ VTexp } \\
\hline$(\mathrm{ml})$ & $493(166)$ \\
\hline$\left(\mathrm{ml} / \mathrm{kg}^{\mathrm{b}}\right)$ & $7.2(3.0)$ \\
\hline $\mathrm{V}^{\prime} \mathrm{E}(\mathrm{l} / \mathrm{min})$ & $12.1(4.7)$ \\
\hline \multicolumn{2}{|l|}{ Leak } \\
\hline$(1 / \min )$ & $3.5(1.2-5.8)$ \\
\hline$(\%)$ & $27(9-55)$ \\
\hline
\end{tabular}

All values mean (SD) or median (25-75 interquartile range)

Leak measured leak in $1 / \mathrm{min}$ and as a $\%$ of $\mathrm{V}^{\prime} \mathrm{E}$; PSL pressure support level; $R R$ patient respiratory rate determined by tracing analysis; $t i_{p}$ patient neural inspiratory time determined from EMGd tracing; $t i_{\text {excess }}$ duration of pressurization by the ventilator in excess of $\mathrm{ti}_{\mathrm{p}} ; V T \exp$ expired tidal volume expressed in $\mathrm{ml}$ and $\mathrm{ml} / \mathrm{kg} ; V^{\prime} E$ minute volume

${ }^{a}$ Expiratory cycling criterion, in \% of peak inspiratory flow

b measured body weight

association was found between leak, auto-triggering, double triggering, premature cycling, or ti $\mathrm{ixcess}_{\text {ex }}$ Multivariate analysis showed that the level of pressure support (OR: 1.32 per additional $\mathrm{cmH}_{2} \mathrm{O}$ of pressure support, $95 \%$ CI: $1.10-1.58 ; P=0.003$ ) and the magnitude of leak (OR: 1.24 per additional $1 / \mathrm{min}$ of leak, 95\% CI: $1.03-$ $1.48 ; P=0.019)$ were associated with the presence of an $\mathrm{AI}>10 \%$. The comfort VAS was higher in patients with an $\mathrm{AI}<10 \%$ (6.5 vs. 5.7, $P=0.027$ ).

No difference was observed in the intubation rate, length of stay in ICU or mortality between patients with or without an $\mathrm{AI}>10 \%$. Only six patients were ventilated with the "NIV mode" turned on: Evita 4 (1), Evita XL (2), Servo I (3). In those six patients, no asynchrony of any type was observed.

Compared to patients in whom NIV succeeded in avoiding intubation, patients who failed had a lower study admission $\mathrm{PaO}_{2}(76 \pm 9$ vs. $88 \pm 6 \mathrm{mmHg}, P=0.02)$ and a higher respiratory rate at admission ( $32 \pm 5$ vs. $27 \pm 3$ breaths/min, $P=0.02)$ and during NIV ( $29 \pm 4$ vs. $24 \pm 3, P=0.01)$.

\section{Discussion}

To our knowledge the present multicenter observational study is the first to document the prevalence and type of asynchronies in patients receiving NIV for acute respiratory failure of mixed etiology. The results show that each specific type of asynchrony was present in 12-23\% of patients, while $43 \%$ of patients presented with severe asynchrony (AI $>10 \%)$. The two factors predictive of the latter were the level of pressure support and the magnitude of leaks.

Before discussing these results, some limitations of the study should be pointed out. First, the tracings were analyzed by only one investigator (L.V.), rather than one from each center, which could lead to systematic bias in their interpretation. However, the methodology had been strictly defined beforehand and tracings on which doubt persisted were discussed among investigators during data session meetings. Furthermore, the random analysis of samples comparing clinical and tracing analysis for ineffective breaths and auto-triggering showed no difference. Still, one cannot exclude that some events were missed or erroneously reported as present. Second, the three participating centers are considered as experienced in the use of NIV. This suggests that if the recordings had been made in centers with less experience with the technique, a higher prevalence of asynchrony might have been documented. Therefore, our figures probably reflect the lower end of the range of prevalence of asynchrony in patients receiving NIV for acute respiratory failure. Third, most patients had already received a few NIV sessions before being included in the study. Had the recordings been made in all patients during the first NIV session, the prevalence and severity of asynchrony would likely have been higher. Fourth, our patient population was fairly modest in number and was heterogeneous both in terms of chronic disease and cause of acute respiratory failure, which might tend to not reflect the specific problems experienced with patients in whom NIV is difficult, e.g., severe COPD or pneumonia. However, the purpose of the study was to "take a picture" of a real-life population of patients in need of NIV, and to that end the various acute and chronic diagnoses seem adequately representative of this situation and are in line with published data $[14,15]$. Finally, an ICU ventilator was used in all patients. This reflects the usual practice of the participating centers, and is in line with other published results [14], mainly because of the higher capacity of these machines to deal with a high inspiratory demand [16]. Nonetheless, our results might not be applicable to the use of a bilevel device, given that these machines deal better with leaks than ICU ventilators when the latter's NIV mode is not activated [17]. In this same line of thought, due to the observational nature of the study, eight different ventilator types were used, which might have influenced the results. However, had only one type of machine been used, asynchrony might have been attributed to this particular type of ventilator, whereas we found no obvious differences in asynchronies between machines. 
Let us now discuss the various asynchronies observed:

Ineffective triggering Ineffective triggering has been shown to increase the work of breathing, most often due to the added inspiratory threshold load associated with dynamic hyperinflation $[18,19]$. Ineffective breaths were present in eight (13\%) of patients. During NIV, one would intuitively expect leaks to increase the occurrence of ineffective efforts, although no clinical study has directly addressed this point. However, in two bench model studies mimicking NIV conditions, leaks were shown to increase the trigger delay [17] and the number of ineffective breaths [20]. This hypothesis is further supported by the fact that in our patients presenting ineffective breaths the magnitude of leak was higher and the VTe lower than those of patients without such wasted efforts (Table 4). Nonetheless, the weak correlation between the magnitude of leaks and the number of ineffective breaths suggests that leaks are not the only cause of such breaths, or that there is no direct quantitative relationship between these two parameters. Intrinsic PEEP has been shown to lead to ineffective efforts in COPD patients [21], but this was not assessed in the present study. In any case, ineffective efforts were not more prevalent or severe in patients with COPD.

Auto-triggering Leaks have been shown to be a major factor leading to auto-triggering [17, 22]. In our eight (13\%) patients in whom auto-triggering was present, the magnitude of leak was higher (Table 4). However, no correlation could be found between leak volume and the severity of auto-triggering. This suggests that once a critical threshold has been reached, above which autotriggering occurs, the frequency of its occurrence depends on several factors such as trigger setting, type of trigger, and design of the ventilator, as shown in a recent bench study [17].

Double-triggering Double triggering is often associated with an insufficient level of pressure support, and results from the same pronounced inspiratory effort retriggering the ventilator after it has discontinued pressurization. In the $9(15 \%)$ patients exhibiting double triggering, the $\mathrm{ti}_{\mathrm{p}}$ was higher and the level of pressure support lower (Table 4). Even though $\mathrm{ti}_{\mathrm{p}}$ was not measured in the study by Thille et al., analysis of the results shows that double triggering was associated with the same imbalance whereby the ventilator's pressurization time is too short in the face of an increased inspiratory demand [10]. This was also the case in our patients, to which an insufficient level of pressure support probably contributed.

Short cycle (premature cycling) Premature cycling occurred in only seven $(12 \%)$ of the patients, and was associated with a longer $\mathrm{ti}_{\mathrm{p}}$, an expected finding. One of the main causes of premature cycling is the presence of

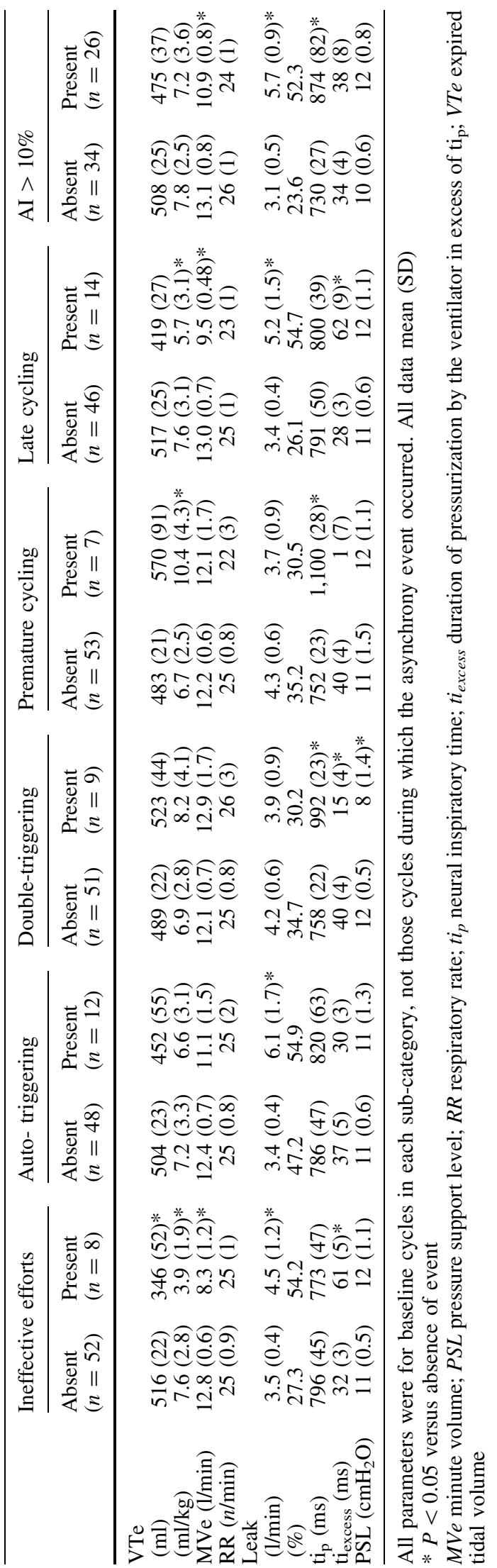


restrictive respiratory mechanics [23, 24]. Interestingly, in our study, acute community-acquired pneumonia was more prevalent in patients in whom premature cycling was present.

Prolonged cycle (delayed cycling) Delayed cycling was present in $14(23 \%)$ of our patients. In intubated patients the main cause of delayed cycling is the presence of obstructive mechanics $[23,25]$. On the other hand, during NIV, while obstructive mechanics are likely to be a contributing factor, leaks have been shown to be a major determinant of delayed cycling $[7,8]$. This was probably also the case in our patients, as suggested by the higher magnitude of leak and lower VTe and minute volume in patients in whom delayed cycling was present (Table 4). Furthermore, among the 14 patients exhibiting delayed cycling, only 5 had documented COPD. The 19 other patients with COPD had no delayed cycling.

In a recent study testing the predictive value of an automatic algorithm in detecting asynchrony, Mulqueeny et al. found that $40 \%$ of their patients recovering from an episode of acute respiratory failure, and receiving NIV with a bilevel device exhibited an $\mathrm{AI}>10 \%$ [26], which is in line with our documented $43 \%$. It is of interest to note that a comparable prevalence of severe asynchrony was observed even though the patients were studied at a later stage in the course of treatment with NIV and the type of ventilator used was different [26]. This might be coincidental or point to one or several factors that are inherent to NIV, such as leaks, or the difficulty of adapting to PSV. In the study by Thille et al., only $25 \%$ of patients had an AI $>10 \%$ [10]. However, the patients were intubated, a situation with very little, if any, leaks. Therefore, it seems reasonable to assume that leaks play a key role in causing many of the asynchrony events documented in our study and that of Mulqueeny et al. [26]. This assumption is in line with the results of the multivariate analysis showing that both the level of pressure support and the magnitude of leaks were associated with an AI $>10 \%$. A higher level of pressure support has been shown to increase the occurrence of leaks [9]. Regarding the level of leaks in our study ( $27 \%$ of MV), it was in line with the range (25-39\%) found in two other studies in patients receiving NIV [27, 28].

In the six patients in whom the "NIV mode" was activated, no asynchrony event was observed. The primary goal of the "NIV mode" is to compensate for the various interferences of leaks with ventilator function, thereby diminishing the incidence of asynchrony events [17]. Given that one of the two main determinants of an $\mathrm{AI}>10 \%$ was the magnitude of leaks, it is reasonable to assume that the "NIV mode" proved effective in those six patients. As the study was not designed to test this hypothesis, however, such a conclusion remains speculative.
The absence of difference in outcome between patients with and without an AI of $10 \%$ might suggest that asynchrony during NIV is of little clinical importance. In our opinion, however, such a conclusion is not warranted at this stage for several reasons. First, the study was not designed to document such an impact. It only looked at the prevalence of asynchrony during one NIV session. Compared to mechanical ventilation in intubated patients which is a continuous process, NIV is applied intermittently. Hence, asynchrony occurring during one NIV session might not be present during the next session, thereby having only a limited impact on NIV failure. Second, the cutoff level of $10 \%$ to define severe asyncrhony, associated with adverse outcome in intubated patients [10], might not be appropriate in the setting of NIV. Third, the cause of asynchrony might vary, e.g., respiratory muscle fatigue or weakness, poor lung mechanics, and agitation in intubated patients versus leaks during NIV, the prognostic significance being quite different. Supporting this view, it has been shown that the nature of the underlying lung disease and absence of arterial blood gas improvement are predictors of NIV failure [29]. In line with these findings, admission $\mathrm{PaO}_{2}$ was lower and respiratory rate at admission and during NIV was higher in patients in whom NIV failed. Most of the time NIV is delivered over a relatively short period of the time and the influence of the early sessions of NIV is probably critical. Indeed, most failures occur within the first 48 hours of treatment. Finally, it is possible that some patients did not require NIV anymore. Indeed, although the patients had received only $4 \pm 2$ prior sessions of NIV, one cannot exclude that some patients could have already been weaned off NIV.

Not surprisingly, patients with an $\mathrm{AI}<10 \%$ had a higher VAS comfort score, which suggests that, irrespective of improving or not improving synchrony impacts outcome, it would at least make the NIV experience more comfortable for patients. Beyond the obvious merit of such an improvement, one must bear in mind that in large epidemiologic study on NIV, patient intolerance to the technique was one of the key factors predicting its failure [4].

\section{Conclusion}

The present observational study documented a prevalence of between $12-23 \%$ of various asynchrony events in a group of patients receiving NIV for acute respiratory failure of various causes. Furthermore, severe asynchrony was observed in $43 \%$ of the patients. Even though the study was not designed to determine the exact cause of asynchrony, several indices point to leaks as a major contributing factor. The "NIV mode", designed to 
attenuate the consequences of leaks on patient-ventilator synchrony, was markedly underused by the staff even though it was available on most of the machines used. These results suggest that clinical research should be NIV. performed to determine if the "NIV mode" actually does reduce the incidence of asynchrony, and if so, efforts should be made to train staff in its use when applying

\section{References}

1. Peter J, Moran J, Hughes J (2002) Noninvasive mechanical ventilation in acute respiratory failure - a metaanalysis update. Crit Care Med 30:555562

2. Liesching T, Kwok H, Hill N (2003) Acute applications of noninvasive positive pressure ventilation. Chest 124:699-713

3. Ferrer M, Esquinas A, Leon M, Gonzalez G, Alarcon A, Torres A (2003) Noninvasive ventilation in severe hypoxemic respiratory failure. Am J Respir Crit Car Med 168:14381444

4. Carlucci A, Richard J, Wysocki M, Lepage E, Brochard L (2001) Noninvasive versus conventional mechanical ventilation. An epidemiologic survey. Am J Respir Crit Care Med 163:874-880

5. Tobin M, Jubran A, Laghi F (2001) Patient-ventilator interaction. Am J Respir Crit Care Med 163:1059-1063

6. Kondili E, Prinianakis G, Georgopoulos D (2003) Patient-ventilator interaction. Br J Anaesth 91:106-119

7. Calderini E, Confalonieri M, Puccio P, Francavilla N, Stella L, Gregoretti C (1999) Patient-ventilator asynchrony during noninvasive ventilation: the role of expiratory trigger. Intensive Care Med 25:662-667

8. Prinianakis G, Delmastro M, Carlucci A, Ceriana P, Nava S (2004) Effect of varying the pressurisation rate during noninvasive pressure support ventilation. Eur Respir J 23:314-320

9. Schettino G, Tucci M, Sousa R, Valente Barbas C, Passos Amato M, Carvalho C (2001) Mask mechanics and leak dynamics during noninvasive pressure support ventilation: a bench study. Intensive Care Med 27:1887-1891

10. Thille A, Rodriguez P, Cabello B, Lellouche F, Brochard L (2006) Patient-ventilator asynchrony during assisted mechanical ventilation. Intensive Care Med 32:1515-1522

11. Brochard L, Mancebo J, Wysocki M, Lofaso F, Conti G, Rauss A, Simonneau G, Benito S, Gasparetto A, Lemaire F, Isabey D, Harf A (1995) Noninvasive ventilation for acute exacerbations of chronic obstructive pulmonary disease. N Engl J Med 333:817-822
12. Hill N, Brennan J, Garpestad E, Nava S (2007) Noninvasive ventilation in acute respiratory failure. Crit Care Med 35:2402-2407

13. Vitacca M, Bianchi L, Zanotti E, Vianello A, Barbano L, Porta R, Clini E (2004) Assessment of physiologic variables and subjective comfort under different levels of pressure support ventilation. Chest 126:851-859

14. Demoule A, Girou E, Richard J, Taille S, Brochard L (2006) Increased use of noninvasive ventilation in French intensive care units. Intensive Care Med 32:1747-1755

15. Schettino G, Altobelli N, Kacmarek R (2008) Noninvasive positive-pressure ventilation in acute respiratory failure outside clinical trials: experience at the Massachusetts General Hospital. Crit Care Med 36:441-447

16. Tassaux D, Strasser S, Fonseca S, Dalmas E, Jolliet P (2002) Comparative bench study of triggering, pressurization and cycling between the home ventilator VPAPII ${ }^{\circledR}$ and three ICU ventilators. Intensive Care Med 28:1254-1261

17. Vignaux L, Tassaux D, Jolliet P (2007) Performance of noninvasive ventilation modes on ICU ventilators during pressure support: a bench model study. Intensive Care Med 33:1444-1451

18. Leung P, Jubran A, Tobin M (1997) Comparison of assisted ventilator modes on triggering, patient effort, and dyspnea. Am J Respir Crit Care Med 155:1940-1948

19. Nava S, Bruschi C, Fracchia C, Braschi A, Rubini F (1997) Patient-ventilator interaction and inspiratory effort during pressure support ventilation in patients with different pathologies. Eur Respir J 10:177-183

20. Fauroux B, Leroux K, Desmarais G, Isabey D, Clement A, Lofaso F, Louis B (2008) Performance of ventilators for noninvasive positive pressure ventilation in children. Eur Respir J. doi: 10.1183/09031936.00144807

21. Nava S, Bruschi C, Rubini F, Palo A, Iotti G, Braschi A (1995) Respiratory response and inspiratory effort during pressure support ventilation in COPD patients. Intensive Care Med 21:871879
22. Bernstein G, Knodel E, Heldt GP (1995) Airway leak size in neonates and autocycling of three flow-triggered ventilators. Crit Care Med 23:17391744

23. Tassaux D, Michotte J, Gainnier M, Gratadour P, Fonseca S, Jolliet P (2004) Expiratory trigger setting in Pressure Support Ventilation: from mathematical model to bedside. Crit Care Med 32:1844-1850

24. Tokioka H, Tanaka T, Ishizu T, Fukushima T, Iwaki T, Nakamura Y, Kosogabe Y (2001) The effect of breath termination criterion on breathing patterns and the work of breathing during pressure support ventilation. Anesth Analg 92:161-165

25. Tassaux D, Gainnier M, Battisti A, Jolliet P (2005) Impact of expiratory trigger setting on delayed cycling and inspiratory muscle workload. Am J Respir Crit Care Med 172:1283-1289

26. Mulqueeny Q, Ceriana P, Carlucci A, Fanfulla F, Delmastro M, Nava S (2007) Automatic detection of ineffective triggering and double triggering during mechanical ventilation. Intensive Care Med 33:2014-2018

27. L'Her E, Deye N, Lellouche F, Taille S, Demoule A, Fraticelli A, Mancebo J, Brochard L (2005) Physiologic effects of noninvasive ventilation during acute lung injury. Am J Respir Crit Care Med 172:1112-1118

28. Lellouche F, Maggiore SM, Deye N, Taille S, Pigeot J, Harf A, Brochard L (2002) Effect of the humidification device on the work of breathing during noninvasive ventilation. Intensive Care Med 28:1582-1589

29. Antonelli M, Conti G, Moro ML, Esquinas A, Gonzalez-Diaz G, Confalonieri M, Pelaia P, Principi T, Gregoretti C, Beltrame F, Pennisi MA, Arcangeli A, Proietti R, Passariello M, Meduri GU (2001) Predictors of failure of noninvasive positive pressure ventilation in patients with acute hypoxemic respiratory failure: a multicenter study. Intensive Care Med 27:1718-1728 